Vol. 42 (1990) [215-229]

\title{
ON LOCAL EQUIVALENCE FOR VECTOR FIELD SYSTEMS
}

\section{P.J. VASSILIOU}

We give sufficient conditions for $C^{\infty}$ vector field systems on $R^{\text {n }}$ with genus $g=1$ to be diffeomorphic to a contact structure. The diffeomorphism is explicitly constructed and used to give the most general integral submanifolds for the systems. Finally the implications of these results for integrable hyperbolic partial differential equations in the plane is discussed.

\section{INTRODUCTION}

The aim of this note is to give some new results on the explicit integration of certain Pfaffian systems with genus $g=1$. The approach adopted is as follows. Given a Pfaffian system $E$ on $\mathrm{R}^{n}$, we ask the question: when is $E$ equivalent to a contact structure with respect to the diffeomorphisms of $\mathbf{R}^{\mathbf{n}}$ and how can an explicit diffeomorphism be constructed? The construction of such a diffeomorphism provides the explicit integral submanifolds for $E$.

The subject of the integration of Pfaffian systems has a long history dating back to 1815 when Pfaff first posed it [10]. Since that time important contributions have been made by a number of authors including von Weber, Goursat, Frobenius, Natani, Clebsch, Grassman, Darboux, and others in the 19th century; (see E. Goursat [5]). In the early 20th century, Cartan's works [3] are well known; less well known is the paper [17] of Vessiot, which contains a number of important results including applications to automorphic systems (see also Pommaret [11] and Kumpera [9]). More recent works are those of Gardner [4] and Bryant [2]. We also note recent applications of the theory of Pfaffian systems to control theory by Hermann $[7,8]$.

The motivation for solving the problem here posed comes from the theory of integrable wave equations. In his study of scalar nonlinear second order partial differential equations in two independent variables, Vessiot [15] showed that whenever the second order characteristics, $\mathcal{M}$, possess two or more invariants and these invariants are taken as independent coordinates, then $\mathcal{M}$ is equivalent to a contact structure on a reduced manifold. This fact allowed Vessiot to set tle the integration problem for these equations in an elegant way. These matters will be discussed briefly in Section 4.

Received 19th October, 1989

The research reported in this paper was carried out while I was a visitor at the Institute of Mathematics and its Applications, the University of Minnesota. It is a pleasure to thank the IMA for its warm hospitality and conducive atmosphere for research. I would also like to thank Peter Olver for a useful discussion of aspects of the results here presented and for pointing out relevant parts of the literature.

Copyright Clearance Centre, Inc. Serial-fee code: 0004-9729/90 \$A2.00+0.00. 


\section{A. The Theory of Pfaffian Systems}

In this section I review briefly some of the known results on Pfaffian systems. Let $T R^{n}$ and $T^{*} R^{n}$ denote the tangent and cotangent bundles on $R^{n}$ respectively. Let $C^{\infty}\left(\mathbf{R}^{n}\right)$ denote the ring of real valued, $C^{\infty}$ functions on $\mathbf{R}^{n}$. Let $\Gamma\left(T^{*}\left(\mathbf{R}^{n}\right)\right)$ denote the $C^{\infty}\left(R^{n}\right)$-module of smooth sections of $T^{*}\left(R^{n}\right)$. A Pfaffian system on $\mathbf{R}^{n}$ is a $C^{\infty}\left(R^{n}\right)$-submodule of $\Gamma\left(T^{*}\left(R^{n}\right)\right)$. Let $\Gamma\left(T\left(R^{n}\right)\right)$ denote the $C^{\infty}\left(R^{n}\right)$-module of smooth sections of $T\left(\mathbf{R}^{n}\right)$. A vector field system on $\mathbf{R}^{n}$ is a $C^{\infty}\left(\mathbf{R}^{n}\right)$-submodule of $\Gamma\left(T\left(R^{n}\right)\right)$. A Pfaffian system $E$ (vector field system $\widetilde{E}$ ) is said to have dimension $r$ at point $x \in \mathrm{R}^{n}$ if there exists a neighborhood $U \ni \boldsymbol{x}$ and $r$-linearly independent 1-forms (vector fields) defined on $U$ which generate $E(\tilde{E})$. I will assume throughout that vector fields systems and Pfaffian systems have constant dimension as $x$ ranges over $U$. Such vector field systems will sometimes be referred to as distributions.

The first important object we may associate to $E$ is a vector field system called the characteristic system of $E$ or the Cauchy system of $E$ denoted char $E$ :

$$
\left.\left.\operatorname{char} E:=\left\{X \in \Gamma\left(T R^{n}\right): X\right\rfloor w=0, X\right\rfloor d w \in E, \text { for all } w \in E\right\}
$$

where $\rfloor$ denotes the interior product. The annihilator of char $E$ is called the Cartan system of $E$ denoted $C(E)$. That is

$$
C(E)=(\operatorname{char} E)^{\perp}
$$

The dimension of the Cartan system of $E$ is an invariant called the class of $E$. The class of $E$ is the minimum number of variables necessary in order to write down local generators for the system (see Gardner [4] and Cartan [3]).

The next important construction is that of the derived map of $E$ which is the $C^{\infty}\left(\mathbf{R}^{n}\right)$-linear map

$$
\pi \circ d=\delta_{E}: \quad E \longrightarrow \Gamma\left(\wedge^{2} T^{*}\left(R^{n}\right)\right) / \Gamma\left(T^{*}\left(\mathbf{R}^{n}\right)\right) \wedge E
$$

obtained by exterior differentiation and then projection into the quotient structure.

Suppose $\delta_{E}$ has constant rank on $\mathbf{R}^{n}$. Define the first derived system $E^{(1)}$ of $E$ by $E^{(1)}=\operatorname{ker} \delta_{E}$. Inductively, define the derived map $\delta_{E^{(i)}}$ of $E$

$$
\delta_{E^{(i)}}: E^{(i)} \longrightarrow \Gamma\left(\wedge^{2} T^{*}\left(\mathbf{R}^{n}\right)\right) / \Gamma\left(T^{*}\left(\mathbf{R}^{n}\right)\right) \wedge E^{(i)}
$$

Then the $(i+1)$-derived system $E^{(i+1)}$ of $E$ is given by

$$
E^{(i+1)}=\operatorname{ker} \delta_{E^{(i)}}
$$


Letting $g_{i}=\operatorname{dim} E^{(i)}$ we have $g_{0} \geqslant g_{i} \ldots$

There must be an $N$ so that $g_{N}=g_{N+1} . N$ defined in this way is called the derived length of $E$. So if $N=0$, then $E^{(1)}=E$ so $\delta_{E}=0$. This means $d(E) \subset E_{\wedge} \Gamma\left(T^{*} R^{n}\right)$, that is, $E$ is Frobenius-integrable. Thus $\delta$ is the zero map when $E$ is integrable so we have the intuitive idea that $\delta$ somehow measures the amount of non-integrability of $E$. The collection $\left(E, E^{(1)}, \ldots, E^{N}\right)$ is called the derived flag of $E$. We have

$$
E^{(N)} \subset E^{(N-1)} \subset \cdots \subset E .
$$

Define integers

We have that

$$
\begin{gathered}
p_{0}=\operatorname{dim} E^{(N)} ; \quad p_{N-i}=\operatorname{dim}\left(E^{(i)} / E^{(i+1)}\right), \\
0 \leqslant i \leqslant N-1 .
\end{gathered}
$$

$$
\operatorname{dim} E=\sum_{i=0}^{N} p_{i} .
$$

If $p_{N+1}$ denotes the codimension of $E$ in $C(E)$ then

$$
\text { class } E=\sum_{i=0}^{N+1} p_{i} .
$$

The Pfaffian system $E$ is said to be of type ( Gardner [4])

$$
\left(p_{0}, p_{1}, \cdot \cdot, p_{N}, p_{N+1}\right) \text {. }
$$

Finally, we mention another important invariant, namely the rank or Engle rank. $E$ has rank $\rho$ if $\rho$ is the smallest integer such that

$$
\alpha_{\wedge}(d \alpha)^{\rho}=0,
$$

for all $\alpha \in E$.

The following theorem is proved in Bryant, Chern and Griffiths [1].

THEOREM 2.1. In a neighborhood of $\mathrm{R}^{n}$ suppose $\alpha$ is a 1-form of rank $\rho$. Then there exists a coordinate system $w_{1}, w_{2}, \ldots w_{n}$, possibly in a smaller neighbourhood such that the form $\alpha$ becomes

$$
d w_{1}+w_{2} d w_{3}+w_{4} d w_{5}+\cdots+w^{2 \rho} d w^{2 \rho+1} .
$$

Interestingly, a proof of this classical theorem is given in [17] using vector fields. The following is also classical and due to von Weber (see [5]). 
Theorem 2.2. Let $E$ be a Pfaffian system of type

and suppose

$$
\begin{gathered}
\left(p_{0}, 1,1,1, \ldots, 1,2\right) \\
\operatorname{dim}\left(C\left(E^{(i)}\right) / E^{(i)}\right)=2, \quad 1 \leqslant i \leqslant N-1,
\end{gathered}
$$

and $E^{(N)}$ is a $p_{0}$-dimensional completely integrable system, then there exist $N+p_{0}+2$ independent functions

$$
\left\{x, z_{1}, \ldots z_{p_{0}}, y, y_{1}, \ldots y_{N}\right\}
$$

such that $E$ is locally generated by

$$
\left\{d z_{1}, d z_{2}, \ldots d z_{p_{0}}, d y-y_{1} d x, d y_{1}-y_{2} d x, \ldots d y_{N-1}-y_{N} d x\right\}
$$

For a proof of this theorem the reader is referred to [2]. Theorems 2.1 and 2.2 were the only local structure theorems known (apart from the case of Frobenius integrable systems) until Bryant gave a new structure theorem ([2], Theorem 2.1).

\section{B. Vector Field Systems}

It will be convenient for what is to follow in Sections 3 and 4 to give some results and definitions for vector field systems. Just as we stated the notion of characteristic vector fields for $E$ we can do so for the dual $\widetilde{E}$. The characteristic system for $\widetilde{E}$, denoted char $\widetilde{E}$ is the set of all $\delta \in \widetilde{E}$ such that $[\delta, \widetilde{E}] \subseteq \widetilde{E}$.

THEOREM 2.3. If $E$ and $\widetilde{E}$ are dual then char $E=$ char $\widetilde{E}$.

Proof: Contraction of forms and fields is defined as follows:

$$
(X\rfloor w)\left(X_{1}, \ldots X_{p-1}\right)=w\left(X, X_{1}, \ldots X_{p-1}\right)
$$

where $X, X_{1}, \ldots X_{p-1}$ are vector fields and $w$ is a $p$-form. We have the identity

$$
\left.\left.\left.d \theta\left(v_{1}, v_{2}\right)=v_{1}\left(v_{2}\right\rfloor \theta\right)-v_{2}\left(v_{1}\right\rfloor \theta\right)-\theta\right]\left(\left[v_{1}, v_{2}\right]\right)
$$

for 1 -forms $\theta$ and vector fields $v_{1}, v_{2}$. So if $\delta \in \operatorname{char} \widetilde{E}$ and $X \in \widetilde{E}$, we have

$$
d \theta(\delta, X)=\delta(X\rfloor \theta)-X(\delta\rfloor \theta)-\theta\rfloor[\delta, X]
$$

and for all $\theta \in E$

$$
d \theta(\delta, X)=(\delta\rfloor d \theta)(X)=\theta][\delta, X]=0
$$

So $\operatorname{char} \widetilde{E} \subseteq$ char $E$. Assuming $\theta \in \operatorname{char} E$ and using $(*)$ then proves the theorem. 
Once again, as for Pfaffian systems, we can define the concept of a derived system for a vector field system. The $i^{\text {th }}$ derived system $\widetilde{E}^{(i)}$ of $\widetilde{E}$ is defined recursively

$$
\widetilde{E}^{(i)}=\widetilde{E}^{(i-1)}+\left[\widetilde{E}^{(i-1)}, \widetilde{E}^{(i-1)}\right], \widetilde{E}^{(0)}=\widetilde{E} .
$$

There will always be an integer $N$ such that

$$
\widetilde{E}^{(N)}=\widetilde{E}^{(N+1)}
$$

meaning that $\widetilde{E}^{(N)}$ is Frobenius integrable.

THEOREM 2.4. For each $i=0,1,2, \ldots N$.

$$
\operatorname{dim} E^{(i)}+\operatorname{dim} \widetilde{E}^{(i)}=n .
$$

Proof: It will be enough to prove that $E^{(i)}$ and $\widetilde{E}^{(i)}$ are dual. Let $x, y \in \widetilde{E}$. Define a set of 1 -forms

$$
\left.\left.\left.\mathfrak{F}=\left\{\theta \in \Gamma\left(T^{*} R^{n}\right) \mid \theta\right] x=\theta\right] y=\theta\right\rfloor[x, y]=0\right\} .
$$

It follows that $\mathfrak{F} \subseteq E$ and that $d \theta(x, y)=0$. Clearly if $\widetilde{E}$ is Frobenius integrable then $\mathfrak{F}=E$. Otherwise we have the maximal subset of $E$ such that $d \theta \in \Gamma\left(T^{*} R^{n}\right)_{\wedge} E$; that is $\theta \in \operatorname{ker} \delta_{E}=E^{(1)}$. This proves that $E^{(1)}$ is the annihilator of $\widetilde{E}^{(1)}$ and the assertion for $i=1$. To prove the result for $i>1$, repeat the argument choosing $x, y \in E^{(i-1)}$ to show that $\left(\widetilde{E}^{(i)}\right)^{\perp}=E^{(i)}$. We need only do this a finite number of times and the theorem is proved.

REMARK. We now have, because of Theorems 2.3 and 2.4,

$$
\begin{aligned}
p_{0} & =n-\operatorname{dim} \widetilde{E}^{(N)} & & \\
p_{N-i} & =\operatorname{dim}\left(\widetilde{E}^{(i+1)} / \widetilde{E}^{(i)}\right), & & 0 \leqslant i \leqslant N-1, \\
\operatorname{dim}\left(\widetilde{E}^{(i)} / \text { char } \widetilde{E}^{(i)}\right) & =\operatorname{dim}\left(C\left(E^{(i)}\right) / E^{(i)}\right), & & 0 \leqslant i \leqslant N-1 .
\end{aligned}
$$

\section{Vector Field Systems, Contact Structures}

\section{AND INTEgRAL SUBMANIFOLDS}

In this section we obtain some results which concern generalisations of the von Weber systems (Theorem 2.2) in terms of the structure of the associated vector field system. Specifically we consider vector field systems $\widetilde{E}$ locally generated in $\mathbf{R}^{(k+1) q+1}$ by

$$
\left\{X, \frac{\partial}{\partial \xi_{1}}, \frac{\partial}{\partial \xi_{2}}, \ldots \frac{\partial}{\partial \xi_{q}}\right\}
$$


and such that the dual Pfaffian system has type

while

$$
\begin{gathered}
(0, q, q, \ldots \ldots, q+1), \\
\operatorname{dim}\left(C\left(E^{(i)}\right) / E^{(i)}\right)=q+1, \quad 1 \leqslant i \leqslant N-1
\end{gathered}
$$

and derived length $N=k$.

We will obtain sufficient conditions in terms of the structure of $\tilde{E}$ in order that $\widetilde{E}$ be diffeomorphic to the $k^{\text {th }}$-order contact structure $\Omega^{k}\left(R, R^{q}\right)^{\perp}$ on $J^{k}\left(R, R^{q}\right)$, the $k^{\text {th }}$ order jet bundle of maps $R \longrightarrow \mathbf{R}^{q}$. Importantly, we will show how the explicit diffeomorphism may be constructed. Notice that when $q=1$, we obtain the von Weber system which is known to be equivalent to the $k^{\text {th }}$ order contact structure $\Omega^{k}(R, R)$ on $J^{k}(R, R)$.

On $J^{k}\left(R, R^{q}\right)$, the $k^{t h}$-order contact structure is locally generated by

$$
\begin{gathered}
\left\{\frac{\partial}{\partial x}+z_{11} \frac{\partial}{\partial z_{10}}+z_{12} \frac{\partial}{\partial z_{11}}+\cdots+z_{1 k} \frac{\partial}{\partial z_{1, k-1}}+z_{21} \frac{\partial}{\partial z_{20}}+\cdots+z_{2 k} \frac{\partial}{\partial z_{2, k-1}}+\cdots\right. \\
\left.+z_{q 1} \frac{\partial}{\partial z_{q 0}}+\cdots+z_{q k} \frac{\partial}{\partial z_{q, k-1}}, \frac{\partial}{\partial z_{1 k}}, \frac{\partial}{\partial z_{2 k}}, \cdots, \frac{\partial}{\partial z_{q k}}\right\} \\
=\left\{J_{k}^{q}, \frac{\partial}{\partial z_{1 k}}, \cdots \frac{\partial}{\partial z_{q k}}\right\} .
\end{gathered}
$$

We seek a diffeomorphism $\psi: R^{(k+1) q+1} \longrightarrow J^{k}\left(R, R^{q}\right)$ such that

$$
\psi_{*} \widetilde{E}=\Omega^{k}\left(\mathbf{R}, \mathbf{R}^{q}\right)^{\perp}
$$

where $\widetilde{E}$ is of the stated type. Equation (3.1) means that there exists $g \in G L(q+1, R)$ such that

$$
\psi_{*}\left(\begin{array}{c}
X \\
\partial / \partial \xi_{1} \\
\vdots \\
\partial / \partial \xi_{q}
\end{array}\right)=g\left(\begin{array}{c}
J_{k}^{q} \\
\partial / \partial z_{1 k} \\
\vdots \\
\partial / \partial z_{q k}
\end{array}\right)
$$

Suppose such a $\psi$ exists. Then

$$
\begin{aligned}
& \psi_{*}(X)=(X x) \frac{\partial}{\partial x}+\sum_{j=1}^{q} \sum_{i=0}^{k}\left(X z_{j i}\right) \frac{\partial}{\partial z_{j i}} \\
& \psi_{*}\left(\partial / \partial \xi_{\mu}\right)=\frac{\partial x}{\partial \xi_{\mu}} \frac{\partial}{\partial x}+\sum_{j=1}^{q} \sum_{i=0}^{k}\left(\frac{\partial z_{j i}}{\partial \xi_{\mu}}\right) \frac{\partial}{\partial z_{j i}}
\end{aligned}
$$


If $g$ has the form

$$
g=\left(\gamma_{\alpha \beta}\right)_{1 \leqslant \alpha, \beta<q+1}
$$

equations (3.2), making use of (3.3) become

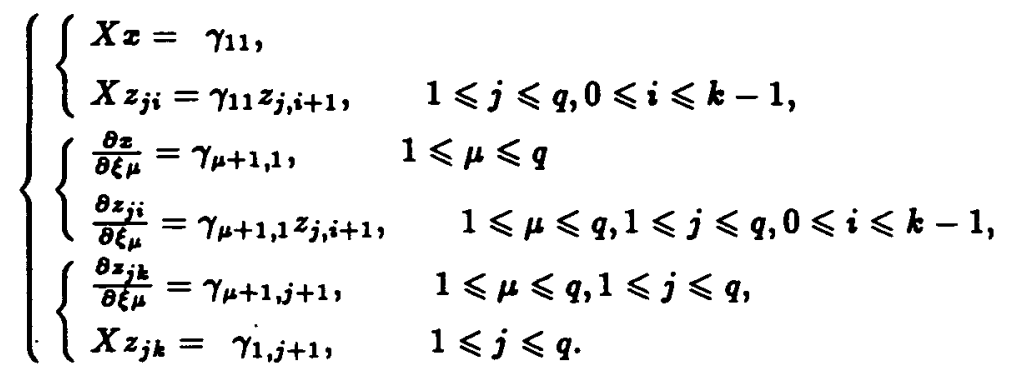

In general the system is overdetermined, the difference between the number of equations and unknowns being

$$
k q^{2}-q-1 \text {. }
$$

Hence in general no diffeomorphism $\psi$ exists. Furthermore, as the equations stand it is not clear how they can be solved in terms of the stated structure conditions. In fact, we restrict ourselves to Pfaffian systems of the stated type only to ensure that the necessary conditions for equivalence are satisfied. In particular, see Hermann ([8], p.138-141) for a discussion of this point. However, since it is enough to seek a particular solution of equations (3.4) we restrict ourselves to diffeomorphisms such that $g$ lies in a subgroup of $G L(q+1, \mathbf{R})$, namely the subgroup generated by the matrices $g \in G L(q+1, \mathbf{R})$ of the form

The equations (3.4) then become

$$
g=\left(\begin{array}{cccc}
\gamma_{11} & \gamma_{12} \ldots & & \gamma_{1, q+1} \\
0 & \gamma_{22} \ldots & & \gamma_{2, q+1} \\
0 & \gamma_{32} \ldots & & \gamma_{3, q+1} \\
\vdots & \vdots & \ldots & \vdots \\
0 & \gamma_{q+1,2} \ldots & & \gamma_{q+1, q+1}
\end{array}\right)
$$
$(A)\left\{\begin{array}{l}X x=\gamma_{11} \\ X z_{j i}=\gamma_{11} z_{j, i+1}\end{array}\right.$
$1 \leqslant j \leqslant q, 0 \leqslant i \leqslant k-1$
(B) $\left\{\begin{array}{l}\frac{\partial x}{\partial \xi_{\mu}}=0 \\ \frac{\theta z_{j i}}{\partial \xi_{\mu}}=0\end{array}\right.$
$1 \leqslant \mu \leqslant q, 1 \leqslant j \leqslant q, 0 \leqslant i \leqslant k-1$
(C) $\frac{\partial z_{j k}}{\partial \xi_{\mu}}=\gamma_{\mu+1, j+1}, X z_{j k}=\gamma_{1, j+1}, 1 \leqslant \mu \leqslant q, 1 \leqslant j \leqslant q$. 
which would seem to make matters worse since the system (3.5) is even more over determined than (3.4). In fact the latter system is manageable.

From the first of equations (3.5)A and (3.5)B we have

$$
\left[\frac{\partial}{\partial \xi_{\mu}}, X\right] x=\frac{\partial \gamma_{11}}{\partial \xi_{\mu}}, \quad 1 \leqslant \mu \leqslant q .
$$

Since we will be content with a particular solution we will impose throughout the condition

$$
\frac{\partial \gamma_{11}}{\partial \xi_{\mu}}=0
$$

and hence $x$ must satisfy

$$
\frac{\partial x}{\partial \xi_{\mu}}=0,\left[\frac{\partial}{\partial \xi_{\mu}}, X\right] x=0
$$

We now impose the further condition that the vector field system

$$
\pi_{0}:\left\{\frac{\partial}{\partial \xi_{\mu}},\left[\frac{\partial}{\partial \xi_{\mu}}, X\right]\right\}_{\mu=1}^{q}
$$

be completely integrable and that therefore there are no integrability conditions to add to (3.7). We also assume that a solution $x$ of (3.7) can be found satisfying $X x \neq 0$.

Consider next the equations

$$
X z_{j 0}=\gamma_{11} z_{j 1}, \frac{\partial z_{j 0}}{\partial \xi_{\mu}}=0,1 \leqslant j \leqslant q .
$$

That is, equations (3.5)A and B with $i=0$. We have that

$$
\left[\frac{\partial}{\partial \xi_{\mu}}, X\right] z_{j 0}=\gamma_{11} \frac{\partial z_{j 1}}{\partial \xi_{\mu}}
$$

and by the assumption that $\gamma_{11} \neq 0$ we have that the condition $\partial z_{j 1} / \partial \xi_{\mu}=0$ is satisfied for all values of $j$ and $\mu$ if and only if $\left[\partial / \partial \xi_{\mu}, X\right] z_{j 0}=0$. Hence the functions $z_{j 0}$ must satisfy the completely integrable system

$$
\frac{\partial z_{j 0}}{\partial \xi_{\mu}}=0,\left[\frac{\partial}{\partial \xi_{\mu}}, X\right] z_{j 0}=0 .
$$

If we now define $z_{j 1}$ by $z_{j 1}=X z_{j 0} / X x$, with $x$ and $z_{j 0}$ defined by equations (3.7) and (3.8) then the partial differential equations for the equivalence map are solved up to $i=0$ and we have also guaranteed that $\partial z_{j 1} / \partial \xi_{\mu}=0$. 
We next proceed to consider the equations (3.5)A and (3.5)B in the case $i=1$. That is

$$
X z_{j 1}=\gamma_{11} z_{j 2}, \frac{\partial z_{j 1}}{\partial \xi_{\mu}}=0 .
$$

Once again because of our choice of $x$ and $z_{j 0}$, we have

$$
\left[\frac{\partial}{\partial \xi_{\mu}}, X\right] z_{j 1}=\gamma_{11} \frac{\partial z_{j 2}}{\partial \xi_{\mu}}
$$

and because $\gamma_{11} \neq 0$ then $\partial z_{j 2} / \partial \xi_{\mu}=0$ if and only if $\left[\partial / \partial \xi_{\mu}, X\right] z_{j 1}=0$ and hence the $z_{j 1}$ must satisfy the completely integrable equations

$$
\frac{\partial z_{j 1}}{\partial \xi_{\mu}}=0,\left[\frac{\partial}{\partial \xi_{\mu}}, X\right] z_{j 1}=0
$$

Now the first equation in (3.9) has already been satisfied because of our choice of $x$ and $z_{j 0}$ so we look at the second equation. Because of $X z_{j 0}=\gamma_{11} z_{j 1}$, the second equation in (3.9) becomes

$$
\left[\frac{\partial}{\partial \xi_{\mu}}, X\right]\left(\frac{1}{\gamma_{11}} X z_{j 0}\right)=0
$$

Now

$$
\left[\frac{\partial}{\partial \xi_{\mu}}, X\right]\left(\frac{1}{\gamma_{11}}\right)=\frac{-1}{\left(\gamma_{11}\right)^{2}}\left[\frac{\partial}{\partial \xi_{\mu}}, X\right] \gamma_{11}=\frac{-1}{\left(\gamma_{11}\right)^{2}}\left[\frac{\partial}{\partial \xi_{\mu}}, X\right] X x .
$$

But since $\left[\partial /\left(\partial \xi_{\mu}\right), X\right] x=0$, we have

$$
\left[\frac{\partial}{\partial \xi_{\mu}}, X\right]\left(\frac{1}{\gamma_{11}}\right)=\frac{-1}{\left(\gamma_{11}\right)^{2}}\left[\left[\frac{\partial}{\partial \xi_{\mu}}, X\right], X\right] x .
$$

Finally, since $\left[\partial /\left(\partial \xi_{\mu}\right), X\right] z_{j 0}=0$, we have that

$$
\begin{aligned}
{\left[\frac{\partial}{\partial \xi_{\mu}}, X\right] z_{j 1}=} & -\frac{\left(X z_{j 0}\right)}{\left(\gamma_{11}\right)^{2}}\left[\left[\frac{\partial}{\partial \xi_{\mu}}, X\right], X\right] x \\
& +\frac{1}{\gamma_{11}}\left[\left[\frac{\partial}{\partial \xi_{\mu}}, X\right], X\right] z_{j 0}
\end{aligned}
$$

Hence by the previous considerations and the formula (3.11) it follows that if $x$ and $z_{j 0}$ are chosen to be invariants of the subdistribution

$$
\left\{\frac{\partial}{\partial \xi_{\mu}},\left[\frac{\partial}{\partial \xi_{\mu}}, X\right],\left[\left[\frac{\partial}{\partial \xi_{\mu}}, X\right], X\right]\right\}_{\mu=1}^{q}
$$


of $\widetilde{E}^{(2)}$ such that $X x \neq 0$ then the equations (3.5)A,B will be solved for all $\mu$ and $j$ and for $i=0$ and 1 and we will also have satisfied

$$
\frac{\partial z_{j 2}}{\partial \xi_{\mu}}=0 \quad \forall j \text { and } \mu .
$$

Let us proceed to (3.5)A,B with $i=2$. That is,

$$
X z_{j 2}=\gamma_{11} z_{j 3}, \quad \frac{\partial z_{j 2}}{\partial \xi_{\mu}}=0 .
$$

Continue with the choices of $x, z_{j 0}, z_{j 1}$ and $z_{j 2}$ as above. As before we must have that

$$
\left[\frac{\partial}{\partial \xi_{\mu}}, X\right] z_{j 2}=0
$$

to guarantee that $\partial z_{j 3} / \partial \xi_{\mu}=0$. Now by virtue of our choice of $x, z_{j 0}$ and $z_{j 1}$ and by a calculation similar to that which led to formula (3.11), we find that (3.12) will be satisfied if now $x$ and $z_{j 0}$ are chosen to be invariants of the subdistribution

$$
\left\{\frac{\partial}{\partial \xi_{\mu}},\left[\frac{\partial}{\partial \xi_{\mu}}, X\right],\left[\left[\frac{\partial}{\partial \xi_{\mu}}, X\right], X\right],\left[\left[\left[\frac{\partial}{\partial \xi_{\mu}}, X\right], X\right], X\right]\right\}
$$

of $\widetilde{E}^{(3)}$ with $X x \neq 0$ and that also all the equations will be solved for $i=0,1$, and 2 .

We may continue this process until $i=k-2$ and find that all equations will be satisfied provided $x$ and $z_{j 0}$ are chosen to be invariants of the subdistribution

$$
\left\{\frac{\partial}{\partial \xi_{\mu}},\left[\frac{\partial}{\partial \xi_{\mu}}, X\right],\left[\left[\frac{\partial}{\partial \xi_{\mu}}, X\right], X\right], \ldots\left[\left[\left[^{(k-1) \text {-times }}\left[\frac{\partial}{\partial \xi_{\mu}}, X\right], X\right], \cdots, X\right]\right\}\right.
$$

of $\widetilde{E}^{(k-1)}$ with $X x \neq 0$. This also guarantees that

$$
\frac{\partial z_{j, k-1}}{\partial \xi_{\mu}}=0
$$

and we define $z_{j k}=\left(X_{j, k-1}\right) /(X x)$.

Finally, we come to the equations (3.5)C. These equations are just definitions of the remaining entries of the group element $g$ and we have therefore, by this process, completely solved the partial differential equations defining the equivalence map. We have therefore proved the following. 
TheOREM 3.1. Let $\widetilde{E}:\left\{X, \partial / \partial \xi_{1}, \partial / \partial \xi_{2} \ldots \partial / \partial \xi_{q}\right\}$ be a $C^{\infty}$-vector field system on $R^{(k+1) q+1}$, of type

$$
(0, q, q, q, \ldots q, q+1) \text {. }
$$

and derived length $k$ and such that

$$
\operatorname{dim}\left(\widetilde{E}^{(i)} / \operatorname{char} \widetilde{E}^{(i)}\right)=q+1, \quad 1 \leqslant i \leqslant k-1 .
$$

Suppose the subdistribution $\pi_{0}:\left\{\partial / \partial \xi_{\mu},\left[\delta / \partial \xi_{\mu}, X\right]\right\} \subset \widetilde{E}^{(1)}$ is completely integrable and the distribution $I I \subseteq \widetilde{E}^{(k-1)}$ generated by

$$
\Pi:\left\{\frac{\partial}{\partial \xi_{\mu}},\left[\frac{\partial}{\partial \xi_{\mu}}, X\right],\left[\left[\frac{\partial}{\partial \xi_{\mu}}, X\right], X\right], \ldots\left[\left[\left[^{(h-1)-\text { times }}\left[\frac{\partial}{\partial \xi_{\mu}}, X\right], X\right], \ldots X\right]\right\}_{\mu=1}^{q}\right.
$$

possesses $q+1$ functionally independent invariants $x$ and $\left\{z_{j 0}\right\}_{j=1}^{q}$ and define the set of functions $z_{j 1}, z_{j 2}, \ldots, z_{j k}, 1 \leqslant j \leqslant q$, by the equations

$$
z_{j 1}=\frac{X z_{j 0}}{X x}, z_{j 2}=\frac{X z_{j 1}}{X x}, \ldots z_{j k}=\frac{X z_{j, k-1}}{X x} .
$$

Then, whenever the set of functions $\left\{x, z_{j 0}, \ldots . z_{j k}\right\}$ so defined are functionally independent, they define a local diffeomorphism

such that

$$
\psi: R^{(k+1) q+1} \longrightarrow J^{k}\left(R, R^{q}\right)
$$

$$
\psi_{*} \widetilde{E}=\Omega^{k}\left(\mathbf{R}, \mathbf{R}^{q}\right)^{\perp}
$$

The means of construction of the integral submanifolds for the Pfaffian systems of the type considered in this paper is now clear. By application of Cartan's theory or otherwise it is not difficult to show that the genus of $\widetilde{E}$ is one. An integral submanifold is therefore a map

such that

$$
\begin{gathered}
\Phi: I \longrightarrow \mathrm{R}^{N}, \quad I \subset \mathbf{R}, \\
\Phi^{*} E=0 .
\end{gathered}
$$

Assuming the map $\psi$ has been found as per Theorem 3.1 we have

$$
\left(\psi^{-1}\right)^{*} E=\Omega^{k}\left(\mathbf{R}, \mathbf{R}^{q}\right)
$$

Hence if $j^{k} f: x \longrightarrow J^{k}\left(\mathbf{R}, \mathbf{R}^{q}\right)$ is the $k$-jet extension of any $C^{k}$ map, $f: \mathbf{R} \longrightarrow \mathbf{R}^{q}$, then

$$
\left(j^{k} f\right)^{*}\left(\psi^{-1}\right)^{*} E=\left(j^{k} f\right)^{*} \Omega^{k}\left(\mathrm{R}, \mathrm{R}^{q}\right)=0 .
$$

Hence

$$
\Phi=\psi^{-1} \circ j^{k f}: R \longrightarrow \mathbf{R}^{N}
$$

is the sought after integral submanifold for $E$ in terms of the (now known) diffeomorphism $\psi$. 
REMARK. In view of the works $[7,8]$ it may be worth mentioning the connection between the result of this paper and control theory. Note that in this section we have been studying Pfaffian systems of the form

$$
d x_{i}-f_{i}\left(t, x_{j}, u_{j}\right) d t
$$

where the $x_{i}$ may be regarded as state variables and the $u_{j}$ as control variables. The algorithm here developed expresses each of the variables $x_{i}(t), u_{j}(t)$ in terms of arbitrary functions and their derivatives thereby "parametrising" the state and control space. This may be compared with the so called "Problem of Monge" (see [5], p.310, [7], p.353).

EXAMPLE. We give a simple example to illustrate the results. On $\mathbf{R}^{7}$ we have the vector field system

$$
\widetilde{E}:\left\{\frac{\partial}{\partial t}+\xi_{1}\left(x_{1} \frac{\partial}{\partial x_{1}}+t x_{2} \frac{\partial}{\partial x_{2}}\right)+\xi_{2}\left(x_{3} \frac{\partial}{\partial x_{3}}+t x_{4} \frac{\partial}{\partial x_{4}}\right), \frac{\partial}{\partial \xi_{1}}, \frac{\partial}{\partial \xi_{2}}\right\} .
$$

$\widetilde{E}$ has type $(0,2,2,3)$ and satisfies $\operatorname{dim}\left(E^{(i)} /\right.$ char $\left.\widetilde{E}^{(i)}\right)=3, i=1,2$, and hence satisfies the necessary conditions for equivalence to the contact structure $\Omega^{2}\left(R, R^{2}\right)^{\perp}$. Therefore calculating the subdistribution II in Theorem 3.1, that is,

$$
\left\{\frac{\partial}{\partial \xi_{\mu}},\left[\frac{\partial}{\partial \xi_{\mu}}, X\right]\right\}_{\mu=1}^{2},
$$

we easily find its three invariants in the form

$$
\begin{aligned}
x & =t, \\
z_{10} & =x_{1}^{t} / x_{2}, \\
z_{20} & =x_{3}^{t} / x_{4} .
\end{aligned}
$$

From the equations of Theorem 3.1, we obtain the remaining variables

$$
\begin{array}{ll}
z_{11}=\frac{x_{1}^{t}}{x_{2}} \ln x_{1}, & z_{21}=\frac{x_{3}^{t}}{x_{4}} \ln x_{3}, \\
z_{12}=\frac{x_{1}^{t}}{x_{2}}\left(\ln x_{1}\right)^{2}+\xi_{1} \frac{x_{1}^{t}}{x_{2}}, & z_{22}=\frac{x_{3}^{t}}{x_{4}}\left(\ln x_{3}\right)^{2}+\xi_{2} \frac{x_{3}^{t}}{x_{4}}
\end{array}
$$

and the map $\psi$ is explicitly found. It is easy to check that $\psi$ is a local diffeomorphism and that $\psi_{*} \widetilde{E}=\Omega^{2}\left(\mathbf{R}, \mathbf{R}^{2}\right)^{\perp}$. Calculating $\psi^{-1}$, we find the integral submanifold for $E$ is given explicitly by equation (3.13):

$$
\begin{aligned}
\Phi: t \longrightarrow & \left(t, \exp \left(f_{1}^{\prime} / f_{1}\right), 1 / f_{1} \exp \left(\frac{t f_{1}^{\prime}}{f_{1}}\right), \exp \left(f_{2}^{\prime} / f_{2}\right), 1 / f_{2} \exp \left(t f_{2}^{\prime} / f_{2}\right)\right. \\
& \left.\frac{f_{1} f_{1}^{\prime \prime}-f_{1}^{\prime 2}}{\left(f_{1}\right)^{2}}, \frac{f_{2} f_{2}^{\prime \prime}-f_{2}^{\prime}}{\left(f_{2}\right)^{2}}\right) \\
& =\left(t, x_{1}, x_{2}, x_{3}, x_{4}, \xi_{1}, \xi_{2}\right)
\end{aligned}
$$


where $f_{1}(t), f_{2}(t)$ are arbitrary $C^{2}$ functions $\mathbf{R} \longrightarrow \mathbf{R}$. It is straight-forward to check that $\Phi^{*} E=0$.

4. Relationship to Integrable Wave Equations and the Work of Vessiot.

As mentioned in the introduction, Vessiot [16] used the equivalence of vector field systems to contact structures to obtain explicit general solutions for integrable nonlinear wave equations; that is, equations of the form

$$
\frac{\partial^{2} u}{\partial x_{1} \partial x_{2}}=f\left(x_{1}, x_{2}, u, \frac{\partial u}{\partial x_{1}}, \frac{\partial u}{\partial x_{2}}\right)
$$

when there exist at least two independent first-integrals on each characteristic. In fact, Vessiot used the algorithm worked out in this paper in the case $k=3$ and $q=1$ but, it seems, did not write out a proof. The details of this construction are as follows. We will consider the Liouville equation as an example but the reader will find many more in Vessiot's paper [15].

Take the Liouville equation in the form

$$
\frac{\partial^{2} u}{\partial x_{1} \partial x_{2}}=e^{u}
$$

There are two sets of characteristics for (4.2) on the submanifold $\varepsilon \subset J^{2}\left(R^{2}, R\right)$ defined by

$$
z_{12}-e^{z}=0 \text {. }
$$

These are given by the one-dimensional solutions of the vector field systems

$$
\begin{aligned}
& { }_{1} \widetilde{\Omega}_{L}^{2}:\left\{\frac{\partial}{\partial x_{1}}+z_{1} \frac{\partial}{\partial z}+z_{11} \frac{\partial}{\partial z_{1}}+e^{z} \frac{\partial}{\partial z_{2}}+z_{2} e^{z} \frac{\partial}{\partial z_{22}}, \frac{\partial}{\partial z_{11}}\right\} \\
& { }_{2} \widetilde{\Omega}_{L}^{2}:\left\{\frac{\partial}{\partial x_{2}}+z_{2} \frac{\partial}{\partial z}+e^{z} \frac{\partial}{\partial z_{1}}+z_{22} \frac{\partial}{\partial z_{2}}+z_{1} e^{z} \frac{\partial}{\partial z_{11}}, \frac{\partial}{\partial z_{22}}\right\}
\end{aligned}
$$

where $x_{1}, x_{2}, z, z_{1}, z_{2}, z_{11}, z_{12}, z_{22}$ are local coordinates on $J^{2}\left(R^{2}, R\right)$ such that $\left(j^{2} u\right)^{*} z_{11}$ $=\left(\partial^{2} u\right) /\left(\partial x_{1} \partial x_{1}\right)$, et cetera. The distributions ${ }_{1} \widetilde{\Omega}_{L}^{2}$ and ${ }_{2} \widetilde{\Omega}_{L}^{2}$ are dual to the characteristic 1-forms listed for example by Goursat [6] in his discussion of Darboux' method for the general solution of the Liouville equation. One easily finds the invariants in the form

$$
{ }_{1} \tilde{\Omega}_{L}^{2}:\left\{\begin{array}{l}
\pi_{1}=x_{2}, \\
\pi_{2}=z_{22}-\left(z_{2}\right)^{2} / 2,
\end{array}{ }_{2} \widetilde{\Omega}_{L}^{2}:\left\{\begin{array}{l}
\pi_{1}=x_{1}, \\
\pi_{2}=z_{11}-\left(z_{1}\right)^{2} / 2 .
\end{array}\right.\right.
$$

Now if we adapt a new coordinate system incorporating the above invariants, say

$$
\varphi: \varepsilon \longrightarrow \mathbf{R}^{7}
$$




$$
\begin{array}{ll}
\mu_{1}=x_{1} & \mu_{3}=z_{22}-\left(z_{2}\right)^{2} / 2 \\
\mu_{2}=x_{2} & \mu_{4}=z_{11}-\left(z_{1}\right)^{2} / 2 \\
\zeta_{0}=z & \zeta_{1}=z_{1} \quad \zeta_{2}=z_{2}
\end{array}
$$

then for example $\varphi_{*}\left({ }_{1} \widetilde{\Omega}_{L}^{2}\right)$ is locally generated by

$$
\left\{\frac{\partial}{\partial \mu_{1}}+\zeta_{1} \frac{\partial}{\partial \zeta_{0}}+\left(\mu_{4}+\zeta_{1}^{2} / 2\right) \frac{\partial}{\partial \zeta_{1}}+e^{\zeta_{0}} \frac{\partial}{\partial \zeta_{2}}, \frac{\partial}{\partial \mu_{4}}\right\}
$$

on the reduced manifold which is locally $R^{5}$. Applying Theorem 3.1 to $\varphi_{*}\left({ }_{1} \widetilde{\Omega}_{L}^{2}\right)$ shows that it is diffeomorphic to the third order contact structure $\Omega^{3}(R, R)^{\perp}$. This reduction however does not by itself give the general solution of (4.2). The reduction is only a partial integration. The extra information one requires is the existence of a nonlinear map linking the integral submanifolds of $\tilde{\Omega}_{L}^{2}$ and ${ }_{2} \widetilde{\Omega}_{L}^{2}$. In his remarkable study [16], Vessiot discovered such a nonlinear map for each scalar integrable equation (4.1) and thereby completely cleared up the integration problem for these equations. Finally, it is worth mentioning that recently Vessiot's results were extended to coupled systems of wave equations [14]. Interestingly, in this case the characteristics are not in general diffeomorphic to contact structures (see also [13]).

\section{REFERENCES}

[1] R.L. Bryant, S.S. Chern, P.A. Griffiths, 'Exterior Differential Systems', in Proceedings of the 1980 Beijing Symposium (Science Press, 1982).

[2] R.L. Bryant, Ph.D. Thesis, University of North Carolina, Chapel Hill (1979).

[3] E. Cartan, Les Systemes Differentiels Exterieurs et leur Applications Geometrique (Hermann, Paris, 1945).

[4] R.B. Gardner, 'Invariants of Pfaffian systems', Trans. Amer. Math. Soc. 126 (1965), 514-533.

[5] E. Goursat, Lecons sur le probleme de Pfaff (Hermann, Paris, 1922).

[6] E. Goursat, Lecons sur l'intégration des équations aux dérivés partielles du second ordre á deux variable independantes, Vol II (Hermann, Paris, 1898).

[7] R. Hermann, 'The theory of equivalence of Pfaffian systems and input systems under feedback', Math. Systems Theory 15 (1982), 343-356.

[8] R. Hermann, 'Invariants for feedback equivalence and Cauchy characteristic multifoliations of nonlinear control systems', Acta. Appl. Math. 11 (1988), 125-153.

[9] A.K. Kumpera, 'Invariants differentiels d'un pseudogroupe de Lie I \& II', J. Differential Geom. 10 (1975), 347-416.

[10] G.F. Pfaff, 'Abh. der K.P.', Akademie der Wissenschaften zu Berlin, (1814-1815), pp. 76-136. 
[11] J.F. Pommaret, Systems of Partial Differential Equations and Lie Pseudogroups (Gordon and Breach, 1978).

[12] P. Vassiliou, 'An exactly integrable hyperbolic partial differential equation and the nilpotent Lie group $\mathrm{G}_{0,15}$ ' (to appear).

[13] P. Vassiliou, 'On the solution of partial differential equations by the method of Darboux' (to appear).

[14] P. Vassiliou, 'Coupled systems of nonlinear wave equations and finite dimensional Lie algebras I \& II', Acta Appl. Math. 8 (1987), 107-147, 149-163.

[15] E. Vessiot, 'Sur les équations aux dérivés partielles du second ordre, $F(x, y, z, p, q, r, s, t)=0$ integrable par la méthode de Darboux', J. Math. Pure et appl. 18 (1939), 1-61.

[16] E. Vessiot, 'Sur les équations aux dérivés partielles du second ordre, $F(x, y, z, p, q, r, s, t)=0$ integrable par la méthode de Darboux', J. Math. Pure et appl. 21 (1942), 1-65.

[17] E. Vessiot, 'Sur l'integration des faisceaux de transformations infinitesimales dans le cas ou, le degree du faisceau étant $n$, celui du faisceau dérivé est $n+1$ ', Annales de l'Ecole Normale Superieure (3) 45 (1928), 189-253.

Institute for Mathematics and its Applications

University of Minnesota

Minneapolis MN 55455

United States of America permanent address:

Faculty of Information Sciences and Engineering

University of Canberra

Belconnen ACT 2616

Australia 